\title{
The Effects of Histone Deacetylase (HDAC) Inhibitors on FASN Intracellular Localization in Cancer Cells
}

\author{
Elizabeth Colvin ${ }^{1}$, Daiqing Liao ${ }^{1}$ \\ University of Florida \\ Faculty mentor: Daiqing Liao, Department of Anatomy and Cell Biology
}

\begin{abstract}
Understanding the mechanisms underlying cancer biology is crucial for discovering novel and effective therapies to improve patient outcome. Increased lipid production is a major metabolic feature in cancer. The fatty acid synthase (FASN) is a key enzyme for lipid synthesis and is upregulated in cancer. Although fatty acid synthesis is generally thought to take place in the cytoplasm, it has been reported that this enzyme also localizes to the nucleus in cancer cells. We hypothesize that the intracellular localization of FASN could be a potential target to decrease lipid synthesis and ultimately halt cell proliferation. Protein acetylation has been shown to regulate protein intracellular localization. We aim to assess the impact that histone deacetylase inhibitors (HDACi) have on the intracellular location of FASN to reduce de novo lipid production and cancer cell proliferation. We have examined intracellular localization of FASN in cells using immunofluorescence microscopy in cancer cells treated with HDACi and did not detect visible HDACi-induced changes in FASN localization. Other mechanisms, such as phosphorylation also regulate FASN. Future studies will examine the effects of kinase inhibitors on FASN intracellular localization. Keywords: Fatty acid synthase, FASN, Histone deacetylase inhibitors, HDACi, Cancer
\end{abstract}

\section{Introduction}

\section{Fatty Acid Synthase Background}

Fatty acid synthase (FASN) is an enzyme responsible for producing fatty acids from precursor molecules of malonyl-CoA and acetyl-CoA to yield palmitate in the cytoplasm of a cell. The production of fatty acids is an essential cellular process for the generation of signaling molecules, energy storage, and cellular membrane synthesis (Lin et al., 2016). Fatty acids are especially vital to cancer cells due to their rapid cellular proliferation, which requires a large number of lipids. Therefore, cancer cells prefer to activate de novo fatty acid synthesis for the generation of signaling molecules rather than exogenous synthesis, which is characteristic of non-cancer cells (Röhrig \& Schulze, 2016). Also, FASN expression is significantly lower in noncancerous cells due to the large number of fatty acids taken in through a person's diet (Madigan et al., 2014). These differences in FASN expression and the importance of lipid synthesis for cancer cell physiology make FASN a potential target for chemotherapy drugs (Lin et al., 2016); however, therapeutic targets and clinical outcomes have been poor due to adverse 
side effects. Therefore, understanding the underlying mechanisms of FASN and its intracellular localization pathways are critical to finding potential successful treatment options (Madigan et al., 2014).

In noncancerous cells, FASN is localized within the cytoplasm to synthesize fatty acids. Within cancerous cells, per the Warburg effect, glycolytic pathways that are used for energy are highly active, providing more substrates for de novo fatty acid synthesis to occur. FASN also utilizes a large amount of NADPH and other reducing molecules during fatty acid synthesis, which potentially helps balance the redox reactions that are happening (Madigan et al., 2014). Research has shown, in prostate cancer cells, that FASN was located within the nucleus rather than its common cytosolic location. This apparent mislocalization affected the prostate cancers carcinogenesis and could potentially be indicative as a marker for a more aggressive disease (Madigan et al., 2014).

Protein acetylation, which is the addition of an acetyl group to lysine residues by histone acetyltransferases (HATs), is a type of posttranslational modification that can alter the conformation of a protein. Acetylation of specific lysine amino acids has been shown to affect the intracellular localization of proteins through changes in the nuclear localization signals (NLS). These changes have caused proteins to mislocalize or accumulate in specific compartments of the cell (di Bari et al., 2006). Histone deacetylases (HDACs) remove the acetyl group from acetyl lysine. Through this enzymatic activity, HDACs can regulate protein intracellular localization. As discussed below, HDACi potently blocks the deacetylation reaction. Therefore, we wanted to explore the impact posttranslational modifications through HDACi had on FASN localization to see if a mislocalization could be detected.

\section{Molecular Structure of FASN}

FASN's structural components have recently been studied to understand the underlying mechanisms for fatty acid synthesis. It has been found that the FASN systems are elaborately structured with enzymatic domains with covalently bound substrates and shuttled by a mobile acyl carrier protein (ACP) (Grininger, 2014). The structural organization of the FASN type I system forms an X-shaped dimeric structure of $540 \mathrm{kDa}$, as seen in Figure 1 (Grininger, 2014). 


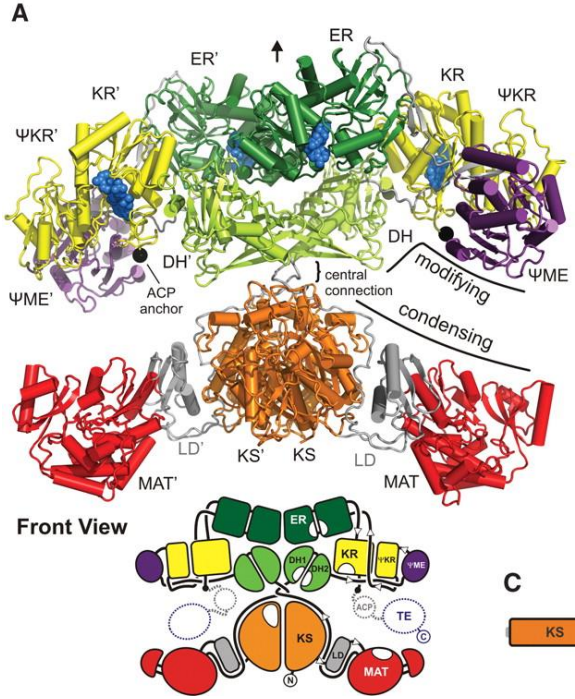

B

'
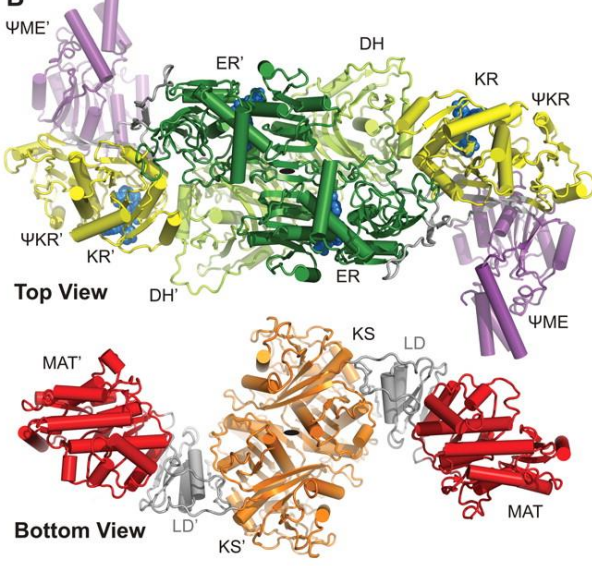

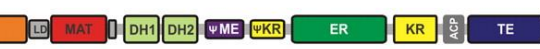

Linear organization

Figure 1. Structural overview of mammalian (pig) FASN protein (Maier, Leibundgut, \& Ban, 2008)

This homodimeric multifunctional polypeptide contains seven catalytic domains in the following order listed linearly from the carboxyl terminus: thioesterase, ACP, $\beta$-ketoacyl reductase, enoyl reductase, $\beta$-hydroxyacyl dehydratase, acetyl/malonyl-CoA transferase and $\beta$ ketoacyl synthase (Flavin, Peluso, Nguyen, \& Loda, 2010). The thioesterase domain has been a therapeutic target for anticancer medications due to its importance in determining the primary product produced, which is palmitate and allows for enzyme turnover. The inhibition of this domain has been shown to halt tumor cell proliferation in mice, but on-going research into structure-based therapeutic agents is necessary (Chakravarty, Gu, Chirala, Wakil, \& Quiocho, 2004).

\section{FASN Transport and Regulation}

FASN is reported to be located in the cytoplasm of cells, but it was also found in the nucleus of prostate cancer (Madigan et al., 2014). For this change in intracellular localization to occur, FASN must be transported across the nuclear envelope. The import to the nucleus and export to the cytoplasm is a complicated process that requires the integration and utilization of multiple proteins (Fu et al., 2018). Due to the large size and polarity of proteins, passive diffusion across membranes rarely occurs. Nuclear pore complexes (NPCs) are needed to cross the nuclear envelope. NPCs are large protein complexes consisting of around 30 proteins called nucleoporins. Macromolecules contain nuclear localization signals and nuclear export signals to control nuclear import or export (Fu et al., 2018). 
For nuclear import of a protein like FASN to occur per the traditional route, the nuclear receptor importin $\alpha / \beta$ is needed. Importin- $\alpha$ recognizes the nuclear localization signal of the protein and heterodimerizes with importin- $\beta$. This complex can then localize to the nuclear envelope where multiple interactions occur to release the protein within the nucleus (Fu et al., 2018). Various shuttling proteins have impacted the intracellular location of proteins in cancer cells. This association between shuttling proteins and cancer may have a decisive role in cancer development and could potentially be a target of chemotherapy drugs (Fu et al., 2018).

Regulation of FASN expression is mediated through multiple transcription factors, especially sterol-regulatory-element-binding protein-1 (SREBP-1), which consists of a family of basichelix-loop-helix-leucine zippers that bind the DNA sequences of the FASN promoter region (Röhrig \& Schulze, 2016). It has been shown that the unique hypoxic microenvironment within solid tumors has led to an increase in SREBP-1 expression, which then increases the transcription of FASN in human breast cancer cells. Although a large amount of information is known about the transcription of FASN, posttranslational modifications affecting FASN are less widely understood. Therefore, a large amount of research is being conducted to measure changes in FASN expression and intracellular location, possibly due to different posttranslational modifications. One modification and a focus in this study is protein lysine acetylation, which can be reversed by histone deacetylases (HDACs). Histone deacetylase inhibitors (HDACi) have been shown to increase FASN acetylation (Lin et al., 2016).

\section{Histone Deacetylase Inhibitors (HDACi)}

Histone acetyltransferases (HATs) and deacetylases (HDACs) are enzymes responsible for the addition and removal of acetyl groups to the amine of lysine to maintain gene expression and cell proliferation. It was previously thought that only histones were acetylated and deacetylated, but new evidence has shown that acetylation can also occur on a multitude of proteins. Acetylation is essential because changes in a cell's ability to properly acetylate have been linked to various diseases, including cancer. Notably, acetylation of nuclear localization signals (NLSs) impacts intracellular localization of a cellular protein. Protein acetylation can be modulated with HDAC inhibitors (HDACi), which have been used in cancer therapy. HDACi allows for an open chromatin conformation, causing the DNA to be more accessible for targeting agents (Suraweera, O'Byrne, \& Richard, 2018). 
HDACi have been investigated as potential therapeutics for cancer treatment due to previous research showing that HDAC protein levels are increased in certain types of cancers. Through the administration of HDACi, acetylation of particular proteins increases. The epigenetic changes have proven to exhibit antitumor activity through inhibition of cell proliferation and apoptosis. Regarding breast cancer, HDACi has proven to be somewhat useful in conjunction with endocrine therapy (Damaskos et al., 2017). Therefore, learning more about the effect HDACi have on cancer cells, and the intracellular localization of FASN can potentially lead to innovative drug treatments.

\section{Methods}

The following procedural outline is commonly utilized for cellular fixation and immunofluorescent (IF) antibody staining in the Liao laboratory. The prostate cancer LNCaP cells were seeded (about 250,000 cells) on glass coverslips in a 6-well plate in $1000 \mu \mathrm{l}$ Dulbecco's Modified Eagle Medium (DMEM) with 10\% fetal bovine serum and penicillin and streptomycin (10 units $/ \mathrm{ml})$. Cells were incubated at $37^{\circ} \mathrm{C}$ for 24 hours. Four hours before cell fixation and antibody staining, HDACi (SR-4370 $(10 \mu \mathrm{M})$ and MS-275 $(3 \mu \mathrm{M}))$ were added.

Fixation began with $1 \mathrm{~mL} 4 \%$ paraformaldehyde (PFA) added to each well for fifteen minutes at room temperature (RT). Cells were then washed and aspirated twice with phosphate buffered saline (PBS). Cells in each well were permeabilized with $1 \mathrm{~mL}$ of $0.2 \%$ Triton for 15 minutes at RT. Cells were washed twice with PBS and then $1 \mathrm{~mL}$ of IF blocking solution (10 mL complete DMEM and $40 \mathrm{~mL}$ of PBS) was added to each well and stood for 30 minutes at RT. Cells were incubated in the dark with the following primary antibody solution for 30 minutes: $320 \mu \mathrm{l}$ of blocking solution plus $0.5 \mu \mathrm{l}$ of anti-FASN antibody (ProteinTech 10624-2-AP, rabbit). After being washed with PBS, cells were incubated again in the dark for 30 minutes with the secondary antibody: $350 \mu \mathrm{l}$ of blocking solution plus $0.5 \mu \mathrm{l}$ of goat anti-rabbit IgG Alexa Fluor 594 (Life Technologies A11012).

Cells were washed with PBS and mounted to glass slides using $25 \mu \mathrm{l}$ of Vectashield, an antifade fluorescent mounting medium with 4',6-diamidino-2-phenylindole (DAPI). A Zeiss microscope was utilized to analyze the intracellular localization of FASN. Ten photos per slide were taken with $20 \mathrm{x}$ (1.25x optivar). The nuclear counterstain created by the mounting medium was exposed to 0.1 seconds of exposure to see the nucleus of each cell. FASN was stained red, 
and images were acquired with 3 seconds of exposure. Photos of each channel were merged and analyzed using Adobe Photoshop.

\section{Results and Discussion}

Upon analysis, we did not detect any notable difference in FASN intracellular localization before treatment with HDACi. Qualitative analysis of slide one, control with serum (Figure 2), and slide two, control without serum (Figure 3), did not show any notable difference in localization or signal intensity of FASN. Therefore, it can be determined that the presence of serum, which contains lipids and growth factors, versus no serum did not play a major role in FASN proliferation or localization.
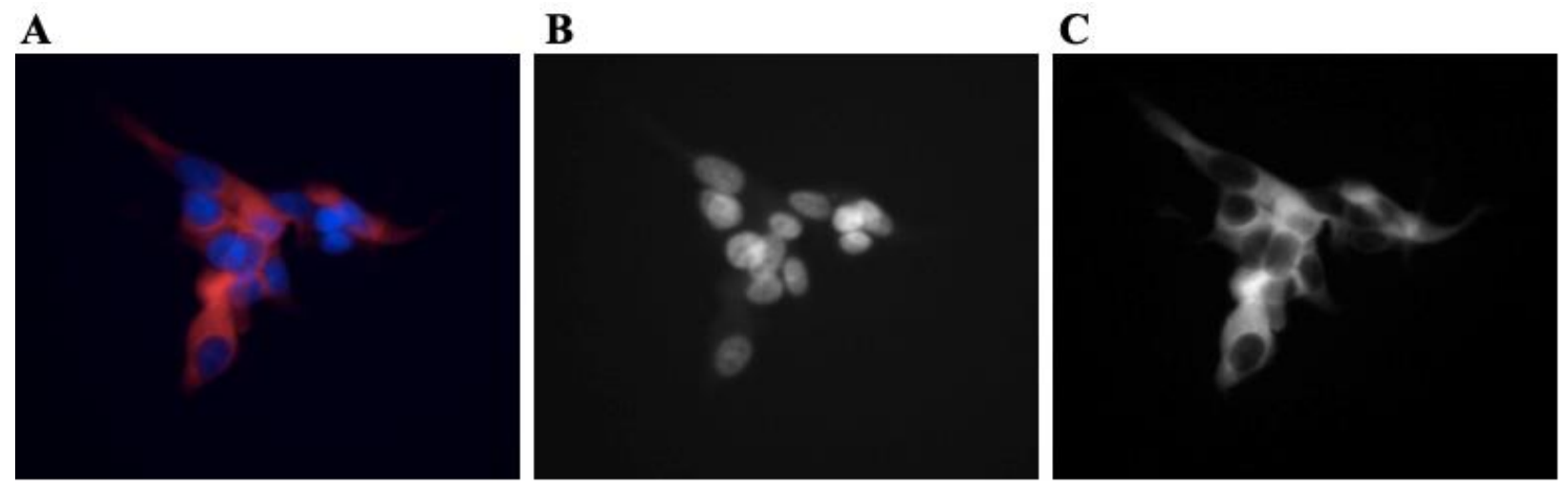

Figure 2. (A) Slide one: cells placed in serum with no HDACi. Presented is a merged photo of the blue and red channels. (B) Blue channel photo of cells in the photo (A) portraying nuclear stain (DAPI). (C) Red channel photo of cells in the photo (A) portraying the fluorescent stain of FASN in the cytoplasm.
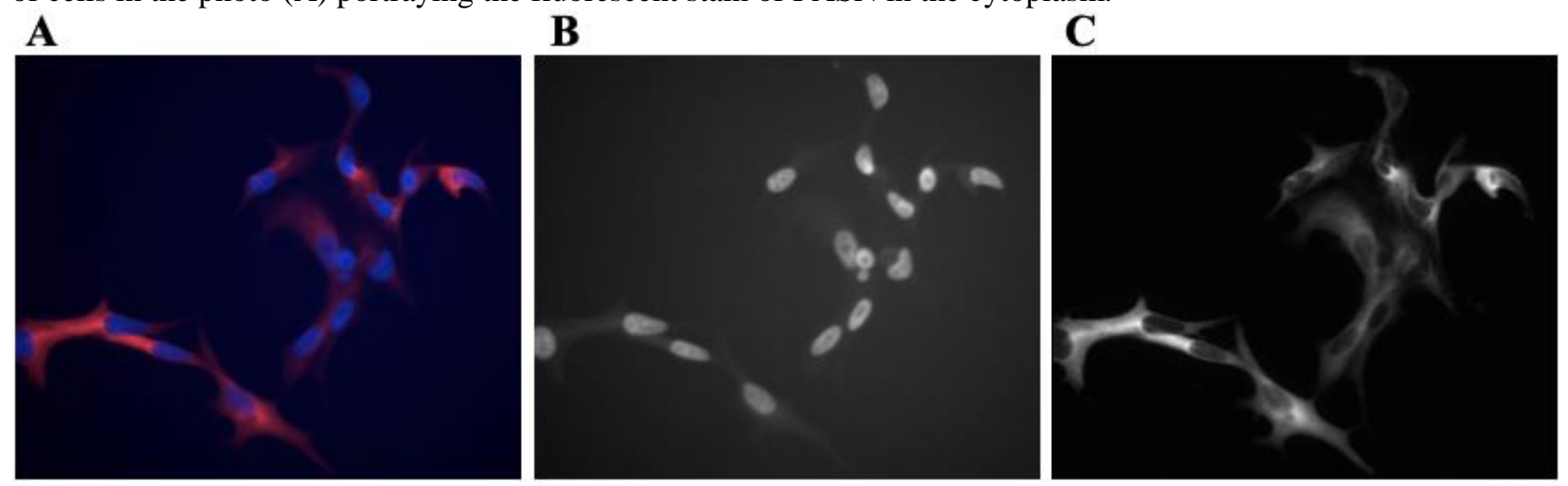

Figure 3. (A) Slide two: cells placed without serum with no HDACi. Presented is a merged photo of the blue and red channel. (B) Blue channel photo of cells in photo (A) portraying nuclear stain (DAPI). (C) Red channel photo of cells in photo (A) portraying the fluorescent stain of FASN in the cytoplasm.

Slides four (Figure 5) and five (Figure 6) appear to have a slightly reduced FASN signal intensity in comparison to slides one (Figure 2) and two (Figure 3). Slide four was treated with 1 
$\mu 1$ DMSO with no serum and slide five was treated with $1 \mu \mathrm{l}$ of $10 \mathrm{mM}$ SR-4370 (final concentration of $10 \mu \mathrm{M}$ ) with no serum. SR-4370 is an HDACi with the chemical structure in Figure 4.

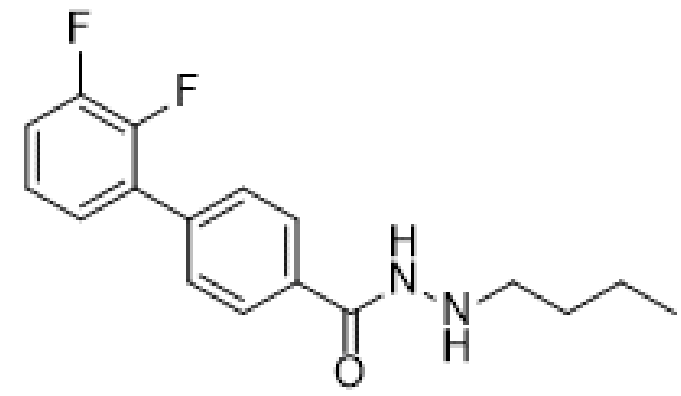

Figure 4. Chemical structure of SR-4370 (Liao, Roush, \& Stowe)

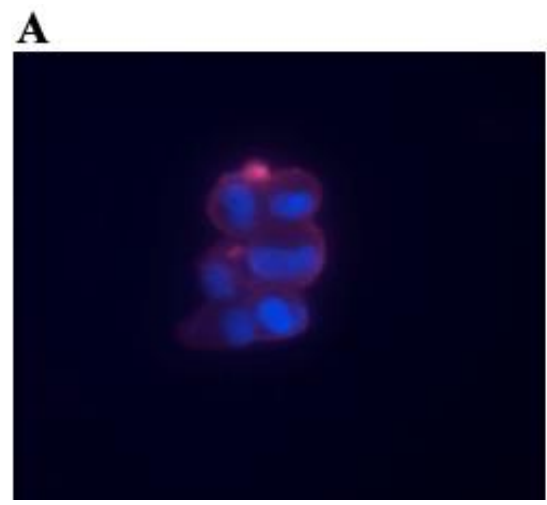

B

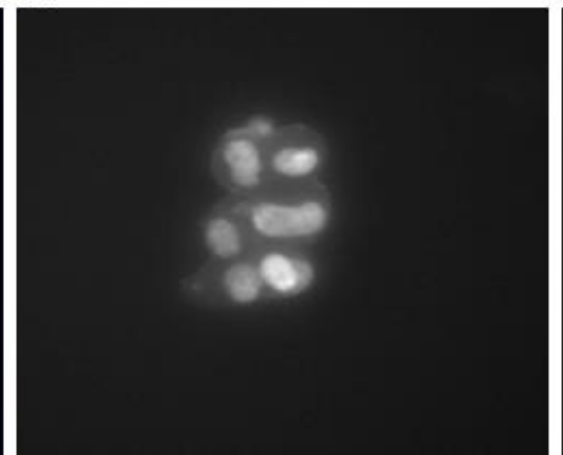

C

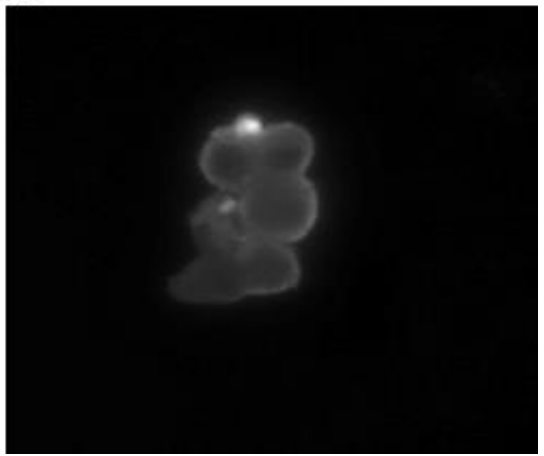

Figure 5. (A) Slide four: cells with no serum and $1 \mu$ DMSO. Presented is a merged photo of the blue and red channel. (B) Blue channel of cells in photo (A) portraying nuclear stain (DAPI). (C) Red channel of slide four cells in photo (A). Presented shows the cytoplasmic localization of FASN with a reduced fluorescent stain in comparison to serum control slides one (Figure 2 above) and two (Figure 3 above).

$\mathbf{A}$

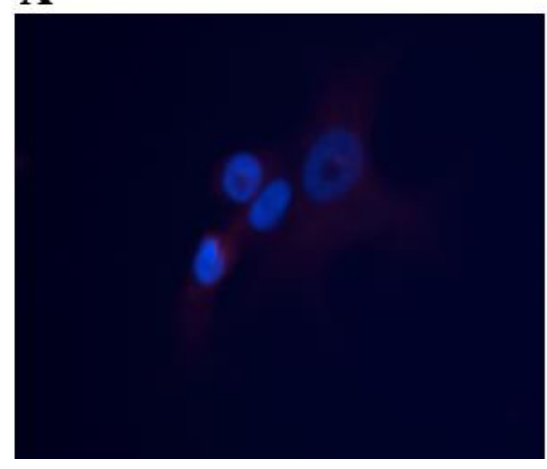

B

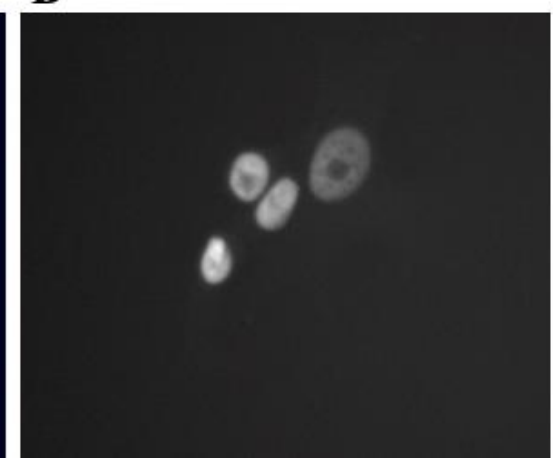

C

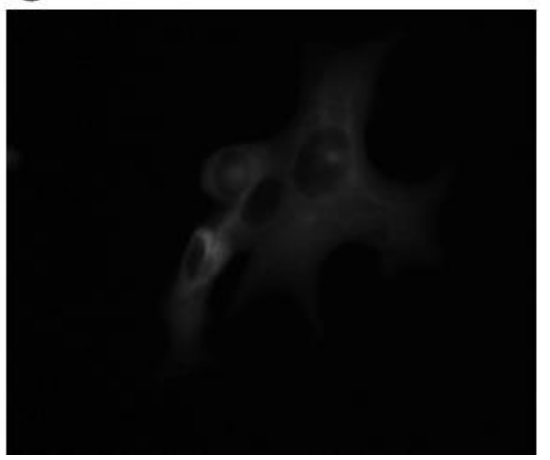

Figure 6. (A) Slide five: cells placed in no serum with HDACi SR-4370. Presented is a merged photo of the blue and red channel. (B) Blue channel of cells in photo (A) portraying nuclear stain (DAPI). (C) Red channel signal of cells in photo (A). This photo shows significantly decreased FASN fluorescent signal compared to DMSO control (Figure 5 above) as well as in cells with or without serum (Figure 2 and three above). 
The slightly decreased signal that was observed in slides four (Figure 5) and five (Figure 6) could potentially indicate a decrease in the FASN expression within the cell. The chosen photos (Figures 5 and 6) are examples of the lowest observed FASN fluorescent signal within their slides. The possibility of human error must also be taken into consideration. An increase in the number of photos taken would help eliminate human error interference; however, taking photos was difficult due to only a few cells left on the slides. This cell line did not adhere well to the slides, and future experiments would benefit from the use of a different cell line.

Slide six showed similar results to slides one through three, with cytoplasmic FASN localization and similar fluorescent signal strength, as seen in Figure 8. Slide six cells were treated with no serum and $1 \mu \mathrm{l}$ of $3 \mathrm{mM}$ MS-275 ( $3 \mu \mathrm{M}$ final concentration), also known as Entinostat, which is an inhibitor of class I HDACs that is utilized in combination with other drugs for advanced hormone receptor-positive breast cancer (Syndax). The structure of MS-275 is shown in Figure 7.

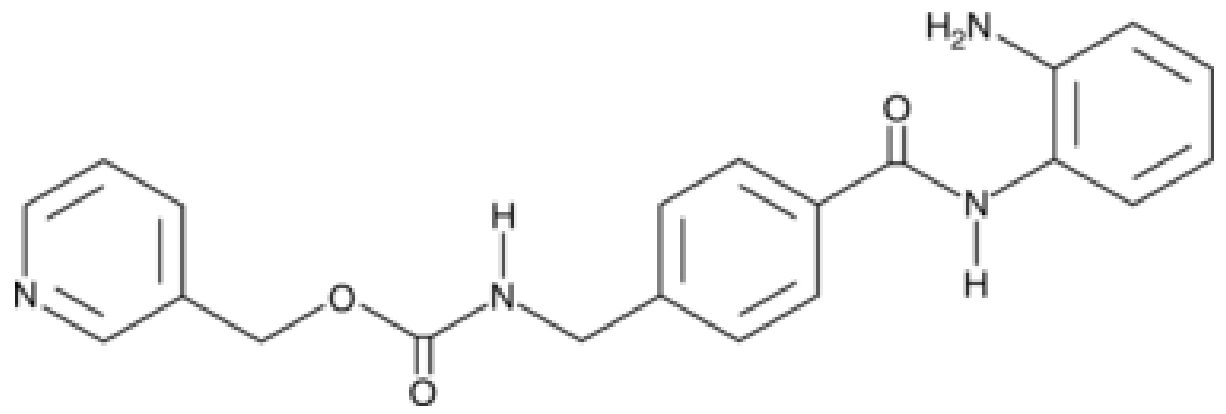

Figure 7. Structure of the HDACi MS-275 (Chemical)

\section{$\mathbf{A}$}

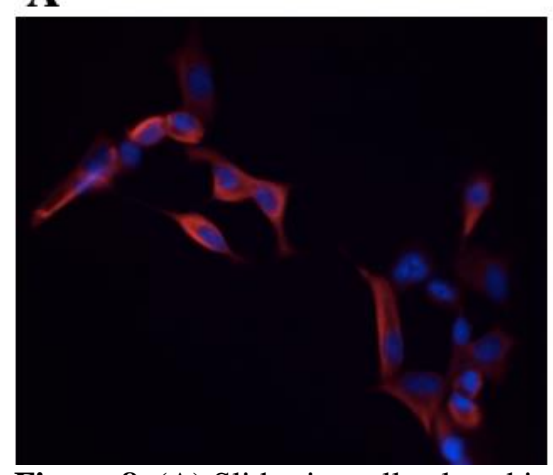

B

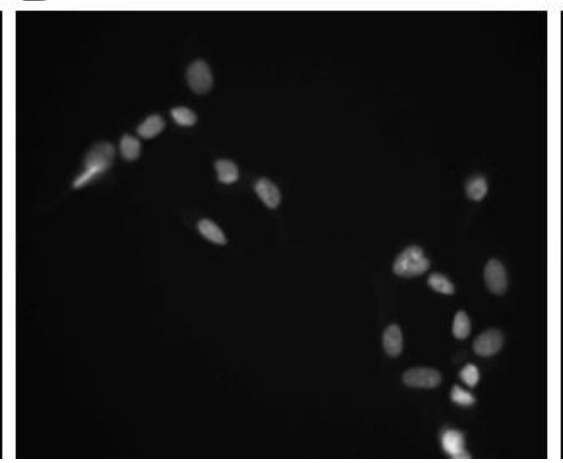

C

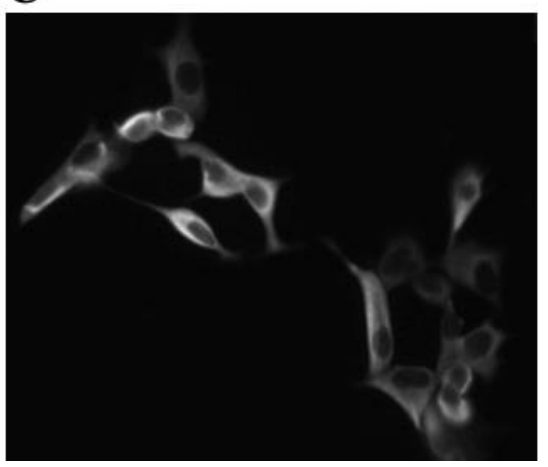

Figure 8. (A) Slide six: cells placed in no serum with $3 \mu \mathrm{M}$ of MS-275. Presented is a merged photo of the Blue red channel. (B) Blue channel of cells in photo (A) portraying nuclear stain (DAPI). (C) Red channel photo of cells in photo (A) portraying the fluorescent stain of FASN in the cytoplasm with a similar fluorescent signal to slides one (Figure 2) and two (Figure 3). 


\section{Conclusion}

Although no dramatic change in the intracellular location of FASN fluorescent signal was observed, there was a decrease in fluorescent signal intensity for slides four, $1 \mu 1$ DMSO with no serum, and five, $10 \mu \mathrm{M}$ SR-4370 with no serum. Utilizing quantitative measures and an increase in trial replication as well as other techniques such as cell fractionation followed by immunoblotting could prove beneficial to yielding more conclusive evidence. Future studies will examine the effects of other enzymes such as protein kinases that may affect the intracellular localization of FASN. Kinase inhibitors and HDACi could also be used in conjunction with one another to examine any potential effects on FASN intracellular localization and protein levels. In addition to FASN, other regulatory proteins, like SREBP-1, can also be analyzed to see if they may have any effect on FASN intracellular localization within cancer cells.

\section{Acknowledgments}

I would like to express my sincere gratitude to Dr. Liao for allowing me this opportunity, guiding me through this process, and mentoring me during my time as an undergraduate. I would also like to thank all of the graduate students within the Liao laboratory that have given me their utmost patience and support during my time researching. Finally, I would also like to show my appreciation for my fellow undergraduate students who have worked with me on this research project and many others.

\section{References}

Chakravarty, B., Gu, Z., Chirala, S. S., Wakil, S. J., \& Quiocho, F. A. (2004). Human fatty acid synthase: structure and substrate selectivity of the thioesterase domain. Proc Natl Acad Sci U S A, 101(44), $15567-15572$.

Chemical, C. MS-275. from https://www.caymanchem.com/product/13284

Damaskos, C., Garmpis, N., Valsami, S., Kontos, M., Spartalis, E., Kalampokas, T., et al. (2017). Histone Deacetylase Inhibitors: An Attractive Therapeutic Strategy Against Breast Cancer. Anticancer Res, 37(1), 35-46.

di Bari, M. G., Ciuffini, L., Mingardi, M., Testi, R., Soddu, S., \& Barilà, D. (2006). c-Abl acetylation by histone acetyltransferases regulates its nuclear-cytoplasmic localization. EMBO Rep, 7(7), 727733.

Flavin, R., Peluso, S., Nguyen, P. L., \& Loda, M. (2010). Fatty acid synthase as a potential therapeutic target in cancer. Future Oncol, 6(4), 551-562.

Fu, X., Liang, C., Li, F., Wang, L., Wu, X., Lu, A., et al. (2018). The Rules and Functions of Nucleocytoplasmic Shuttling Proteins. Int J Mol Sci, 19(5). 
Grininger, M. (2014). Perspectives on the evolution, assembly and conformational dynamics of fatty acid synthase type I (FAS I) systems. Curr Opin Struct Biol, 25, 49-56.

Liao, D., Roush, W. R., \& Stowe, R. L. SR-4370. from https://medkoo.com/products/18841

Lin, H. P., Cheng, Z. L., He, R. Y., Song, L., Tian, M. X., Zhou, L. S., et al. (2016). Destabilization of Fatty Acid Synthase by Acetylation Inhibits De Novo Lipogenesis and Tumor Cell Growth. Cancer Res, 76(23), 6924-6936.

Madigan, A. A., Rycyna, K. J., Parwani, A. V., Datiri, Y. J., Basudan, A. M., Sobek, K. M., et al. (2014). Novel nuclear localization of fatty acid synthase correlates with prostate cancer aggressiveness. Am J Pathol, 184(8), 2156-2162.

Maier, T., Leibundgut, M., \& Ban, N. (2008). The crystal structure of a mammalian fatty acid synthase. Science, 321(5894), 1315-1322.

Röhrig, F., \& Schulze, A. (2016). The multifaceted roles of fatty acid synthesis in cancer. Nat Rev Cancer, 16(11), 732-749.

Suraweera, A., O'Byrne, K. J., \& Richard, D. J. (2018). Combination Therapy With Histone Deacetylase Inhibitors (HDACi) for the Treatment of Cancer: Achieving the Full Therapeutic Potential of HDACi. Front Oncol, 8, 92.

Syndax. Entinostat. from https://www.syndax.com/pipeline/entinostat/ 